\title{
Comparative metabolic profiling of posterior parietal cortex, amygdala, and hippocampus in conditioned fear memory
}

Yoonjeong Jeon ${ }^{1,2}$, Yun Lim ${ }^{1}$, Jiwoo Yeom ${ }^{1,2}$ and Eun-Kyoung Kim ${ }^{1,2^{*}}$

\begin{abstract}
Fear conditioning and retrieval are suitable models to investigate the biological basis of various mental disorders. Hippocampus and amygdala neurons consolidate conditioned stimulus (CS)-dependent fear memory. Posterior parietal cortex is considered important for the CS-dependent conditioning and retrieval of fear memory. Metabolomic screening among functionally related brain areas provides molecular signatures and biomarkers to improve the treatment of psychopathologies. Herein, we analyzed and compared changes of metabolites in the hippocampus, amygdala, and posterior parietal cortex under the fear retrieval condition. Metabolite profiles of posterior parietal cortex and amygdala were similarly changed after fear memory retrieval. While the retrieval of fear memory perturbed various metabolic pathways, most metabolic pathways that overlapped among the three brain regions had high ranks in the enrichment analysis of posterior parietal cortex. In posterior parietal cortex, the most perturbed pathways were pantothenate and CoA biosynthesis, purine metabolism, glutathione metabolism, and NAD ${ }^{+}$dependent signaling. Metabolites of posterior parietal cortex including 4'-phosphopantetheine, xanthine, glutathione, ADP-ribose, ADPribose 2'-phosphate, and cyclic ADP-ribose were significantly regulated in these metabolic pathways. These results point to the importance of metabolites of posterior parietal cortex in conditioned fear memory retrieval and may provide potential biomarker candidates for traumatic memory-related mental disorders.
\end{abstract}

Keywords: Conditioned fear memory, Metabolomics, Posterior parietal cortex, Amygdala, Hippocampus

\section{Introduction}

The increase in the prevalence of mental illnesses has prompted extensive studies aimed at understanding their etiology and pathophysiology, and improving treatment $[1,2]$. People exposed to various traumatic situations are at a great risk for developing several psychiatric conditions including anxiety, depression, and post-traumatic stress disorder (PTSD) [3, 4]. Aversive experiences of stimuli found in a specific situation set the occasion for robust fear of environmental contexts or cues paired with

\footnotetext{
*Correspondence: ekkim@dgist.ac.kr

${ }^{1}$ Department of Brain and Cognitive Sciences, Daegu Gyeongbuk

Institute of Science and Technology (DGIST), Daegu 42988, Republic of Korea

Full list of author information is available at the end of the article
}

these events in humans and other animals [5, 6]. Many aspects of traumatic memories such as fear memory can be studied using Pavlovian fear conditioning paradigms, in which animals learn to associate a conditioned stimulus (CS) including contexts (chamber, cage, etc.) and cues (light, tone, odor, etc.) with an unconditioned stimulus (US) such as a mild foot shock [7-9]. After training, the cue and the context in which an animal was exposed can produce a fear response such as freezing behavior [10].

To understand learning processes including fear conditioning, retrieval, extinction, and renewal, the neural mechanisms by which CS and US representations are encoded in the brain have been investigated. It is well established that the context is processed within the hippocampal-cortical networks, whereas the cue and US 
are processed within the basolateral amygdala (AMG), whose output contributes to conditioned fear [11-14]. In fear learning processes, direct projections from the hippocampus (HPC) to AMG and indirect projections between the HPC and AMG via the prefrontal cortex (PFC) mediate fear responses [15-17]. Especially, ventral hippocampal CA1 projections to the basal AMG are necessary for encoding and retrieval of contextual fear memory [18]. Medial PFC is gradually involved in remote fear memory retrieval [19]. Although the AMG, HPC, and PFC are known as key regions for the regulation of conditioned fear memory, other brain regions that mediate CS-dependent modulation of the fear response have not been fully investigated.

The posterior parietal cortex (PPC) receives various sensory and cognitive stimuli, integrates multisensory signals [20-23], processes sensory information, and transforms this information into behavioral activity [24-26]. Cognitive processes are affected by the response of PPC neurons [27, 28]. Additionally, PPC neurons are activated in contextual fear conditioning and reactivated by fear memory retrieval [29]. These studies support the idea that the PPC has roles in memory formation and processing.

Metabolomics, a qualitative and quantitative analysis of small molecules present in biological samples, has been increasingly used as a tool to discover and develop biomarkers [30-32]. A number of attempts have been made to identify biomarkers of traumatic memory-related mental disorders. For example, using metabolic profiling of prefrontal extracellular fluid, metabolites such as xanthurenic acid, glucose-1-phosphate, sarcosine, and spermidine have been found to predict long-term PTSD-like symptoms [33]. In a study of social defeats (used as a conditioned fear model), the levels of cystine which is converted to cysteine, and fumarate were increased in the nucleus accumbens of stress-resistant and dominant animals, respectively [34]. In the PFC, the level of methionine was also elevated in dominant animals [34]. These studies suggest these metabolites as therapeutic biomarkers of stress-related mental disorders. However, potential biomarkers in other mental diseases remain unidentified. Therefore, identification of several neurochemicals in various brain tissues in the conditioned fear response could provide novel biomarkers that could be used to improve the treatment of psychopathologies associated with mental disorders [34, 35].

Here, we performed an unbiased metabolomics screening in the PPC, AMG, and HPC and identified metabolites relevant to conditioned fear memory. Our data demonstrated that the trends of altered metabolites and metabolic pathways in PPC and AMG were more similar to each other than that of HPC. Moreover, we found that several PPC-specific metabolites significantly changed after the retrieval of fear memory. These results may provide potential biomarker candidates for the diagnosis, risk assessment, and prevention of fear memory-related mental disorders via further validation.

\section{Results \\ Contextual and cued fear conditioning, and retrieval test for mice}

We first used a fear-conditioning paradigm to examine metabolic changes in brain regions induced by fear memory retrieval. Mice were subjected to a fear-conditioning task in which they learned to associate a cue (tone) with a US (foot shock) in a context. Mice in the fear-conditioning (FC) group were first exposed to six pairings of the tone and foot shock. Mice were then placed in the same context and received the tone twice without the foot shock during the next day. Control mice were exposed to the same task as the FC group but did not receive any foot shocks (Fig. 1a). During the task, conditioned fear responses were measured on the basis of the duration of freezing behavior (Fig. 1b, c).

Significant differences in freezing behavior were found between the control and FC groups during fear conditioning on day 1 [time $\times$ task interaction, $\mathrm{F}(6,186)=51.47$, $p<0.0001$; time, $\mathrm{F}(6,186)=49.30, p<0.0001$; task, $\mathrm{F}(1$, $31)=107.9, p<0.0001$, two-way repeated-measures analysis of variance (ANOVA) (Fig. 1b)]. On day 2, significant differences in fear responses to the tone and context were found between the control and FC groups during the retrieval test [time $\times$ task interaction, $\mathrm{F}(2,62)=8.67$, $p=0.0005$; time, $\mathrm{F}(2,62)=9.79, p=0.0002$; task, $\mathrm{F}(1$, $31)=135.6, \quad p<0.0001, \quad$ two-way repeated-measures ANOVA (Fig. 1b)].

In the retrieval test, the levels of context-dependent freezing behavior were measured for the first $300 \mathrm{~s}$ (context test). To determine further changes in freezing by tone in the retrieval test, mice were then exposed to two tones in the same context. The level of freezing behavior was significantly higher in the FC group than the control group in the context only, and context and tone stimuli. After exposure to the tone with the same context, the levels of freezing behavior increased significantly in the FC group in comparison with the context test only (Fig. 1c).

\section{Metabolic profiling of three fear memory-related brain regions}

To identify metabolites specific to conditioned fear memory in each brain region, we performed untargeted metabolite profiling of PPC, AMG, and HPC samples and focused on differences between the FC and control groups. In the three selected brain regions, partial least squares-discriminant analysis (PLS-DA) showed the 


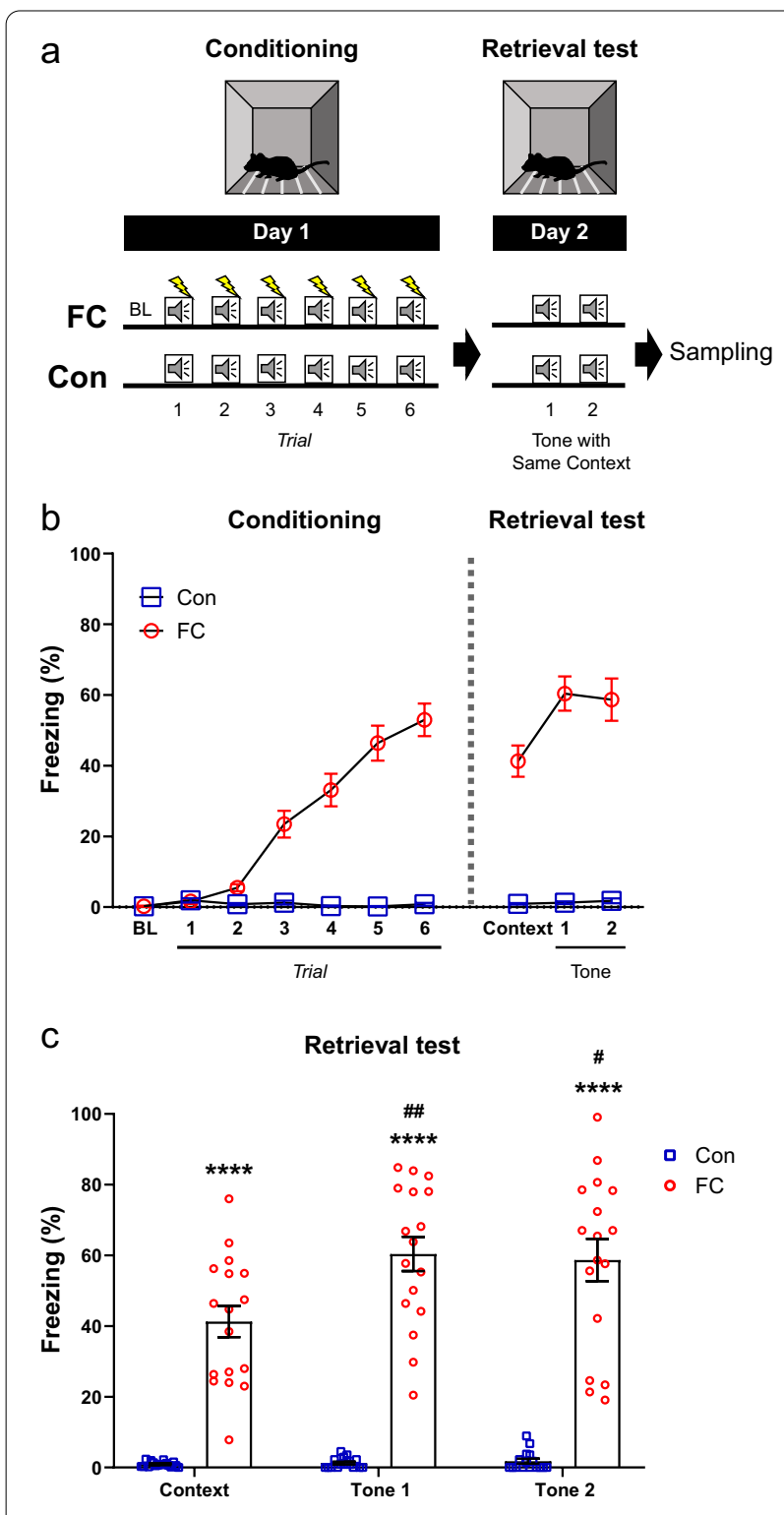

Fig. 1 Behavior changes during fear conditioning and retrieval test. a Schematic diagram showing the experimental procedure for fear conditioning and retrieval test. b Percentage of freezing behavior across the conditioning and retrieval test sessions. Each dot represents the level of freezing when the tone stimulus was presented, except the first dot of each session, which shows the pre-tone baseline and the context test, respectively (Con, $n=16$ mice; $F C, n=17$ mice; two-way repeated-measures ANOVA, data are mean $\pm S E M)$. $\mathbf{c}$ Freezing in the context and tone stimulus during the retrieval test (Con-Context, $\mathrm{n}=16$ mice; FC-Context, $\mathrm{n}=17$ mice; Con-Tone $1, n=16$ mice; FC-Tone $1, n=17$ mice; Con-Tone $2, n=16$ mice; FC-Tone 2, $\mathrm{n}=17$ mice; one-way ANOVA, data are mean \pm SEM; ${ }^{* * * *} p<0.0001$ vs. Con, ${ }^{\#} p<0.05,{ }^{\# \#} p<0.01$ vs. Context). $B L$ baseline, Con control, Context context test, $F C$ fear conditioning variance in the data among five components (Fig. 2). In PLS-DA of PPC, $36.2 \%$ of the variance was explained by Component $1,10 \%$ by Component $2,10.1 \%$ by Component $3,9.1 \%$ by Component 4 , and $5.9 \%$ by Component 5 (Fig. 2a). In the AMG dataset, 27.2\% of the variance was explained by Component $1,15.8 \%$ by Component 2 , $6.7 \%$ by Component $3,6.2 \%$ by Component 4 , and $3.8 \%$ by Component 5 (Fig. 2b). In HPC, 37.4\% of the variance was explained by Component $1,10.9 \%$ by Component 2 , $5.2 \%$ by Component $3,4.5 \%$ by Component 4 , and $3.3 \%$ by Component 5 (Fig. 2c). The score plot for each brain region between Components 1 and 2 is shown in Fig. 2 . PLS-DA indicated nearly perfect separations of FC and control groups in all brain regions. 3D score plots of the PLS-DA of PPC, AMG, and HPC are shown in Additional file 1: Fig. S1.

To identify metabolites driving the separation between the FC and control groups, metabolites from each brain tissue were ranked by variable importance in projection (VIP) scores generated from the PLS-DA. Metabolites with high VIP scores $(\geq 1.0)$ were considered contributing to observed separation; 18 fear memory-relevant metabolites with important variations were identified by ascribing VIP scores in PPC, 25 in AMG, and 29 in HPC. The VIP scores of metabolites in each brain region and the patterns of their changes are shown in Fig. 3a-c. On the basis of the VIP score $\geq 1.0$, some overlapping metabolites tended to be increased and some tended to be decreased ( $p$-value $<0.1$, fold change $\geq|1.1|$ ) after fear retrieval in PPC, AMG, and HPC, as revealed by intersection analysis. Twenty-two metabolites were identified in more than one brain region. Fifteen metabolites differed between FC and control-group mice in both PPC and AMG, 8 in PPC and HPC, and 7 in AMG and HPC. Four metabolites overlapped among all three selected brain regions (Fig. 3d). A heat map in Fig. 3e illustrates the trends of changes in the relative levels of the 65 metabolites compared between $\mathrm{FC}$ and control groups in PPC, AMG, and HPC. Of note, 13 of the 15 metabolites overlapping between PPC and AMG had similar patterns of increases and decreases (Fig. 3e). The detailed data of metabolites such as fold changes and overlaps are provided in Additional file 2: Dataset S1.

\section{Metabolic pathway analysis in conditioned fear memory and identification of representative metabolites in PPC}

To further determine the biological significance of the fear memory retrieval-relevant metabolites in each brain region, we performed a metabolite set enrichment analysis. Metabolites that were significantly changed were selected and the metabolic pathways that involve these metabolites were examined by enrichment analysis. Metabolites with $p$-values $<0.05$ were selected for 

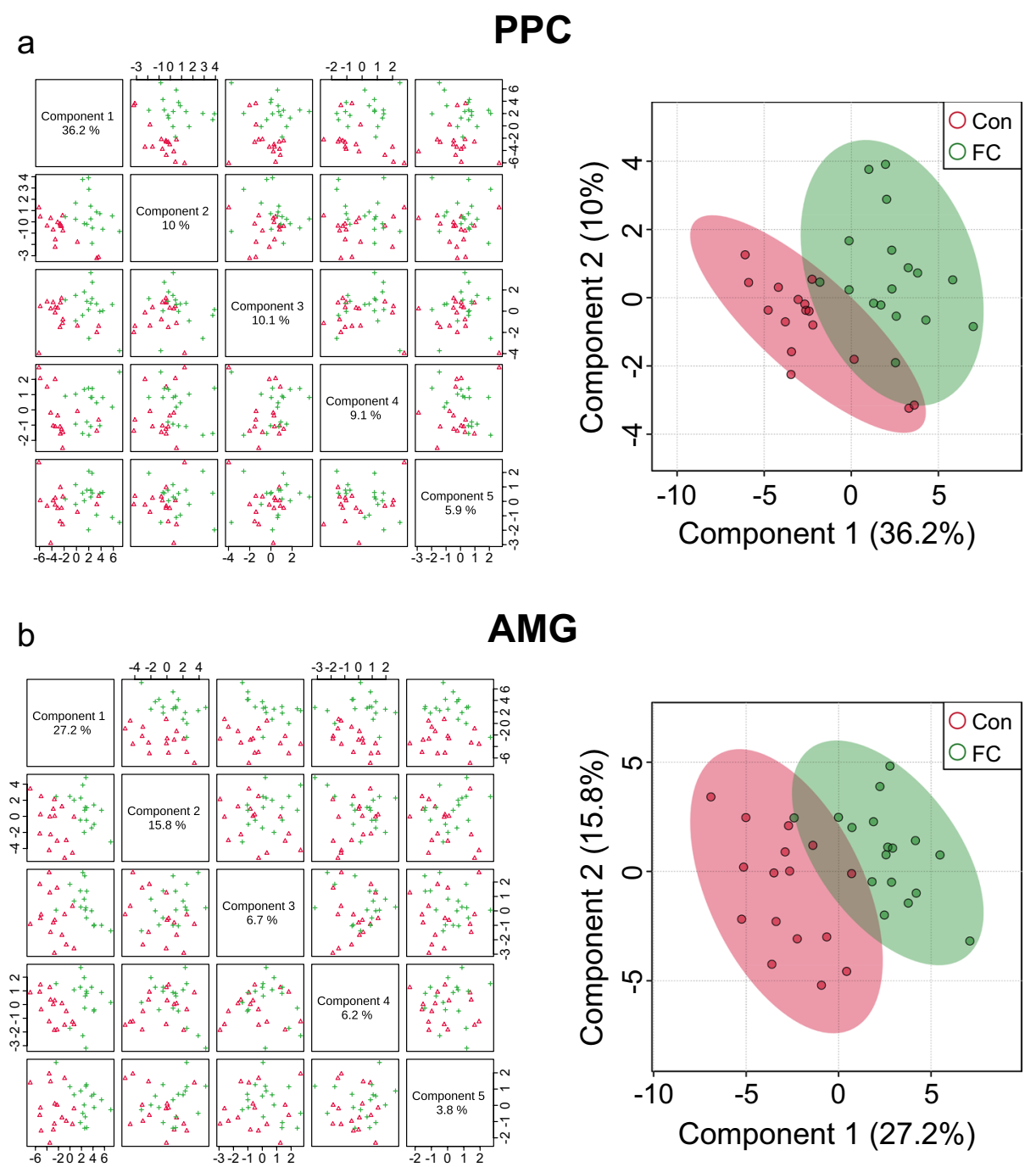

C

HPC
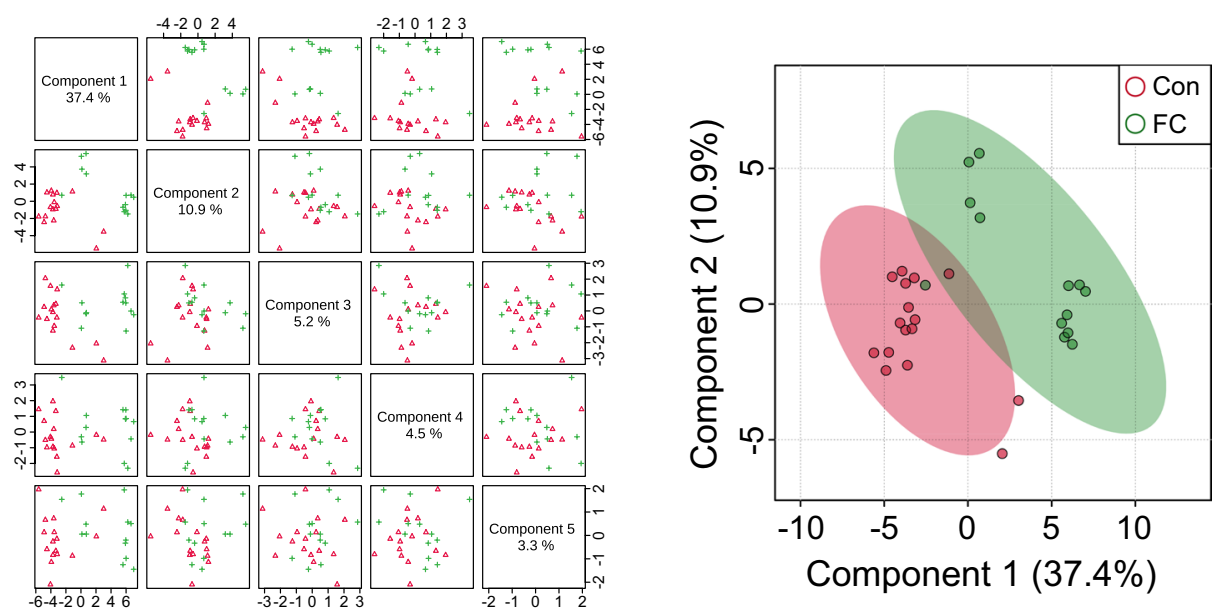

Fig. 2 Pairwise score plots of selected components and 2D score plots in PLS-DA. a PPC. b AMG. c HPC. Each point corresponds to one mouse; shaded ellipses represent 95\% confidence intervals (Con-PPC, $n=16$ mice; FC-PPC, $n=17$ mice; Con-AMG, $n=16$ mice; FC-AMG, $n=17$ mice; Con-HPC, $n=16$ mice; FC-HPC, $n=13$ mice). Con control, FC fear conditioning 
(See figure on next page.)

Fig. 3 Metabolites selected on the basis of VIP score in fear retrieval. a Top metabolites (VIP score $\geq 1.0$ ) in PPC. b Top metabolites (VIP score $\geq 1.0$ ) in AMG. c Top metabolites (VIP score $\geq 1.0$ ) in HPC. $\mathbf{d}$ Venn diagram showing the numbers of overlapping metabolites in each comparison. e Heat map of 65 metabolites selected on the basis of VIP score from the three brain regions. Rows and columns represent the brain regions and the 65 selected metabolites, respectively. Each cell is colored based on log 2 scale of the relative fold change in each brain region. Con control, FC fear conditioning, VIP variable importance in projection

the enrichment analysis (26 in PPC, 35 in AMG, and 43 in HPC). Additionally, metabolites with $p$-values $<0.1$ (10 in PPC, 14 in AMG, and 14 in HPC) were included in the list for the enrichment analysis. Thus, the datasets included 36 metabolites for PPC, 49 for AMG, and 57 for HPC. Enrichment analyses using these datasets identified the top 25 metabolic pathways contributing to the separation between FC and control groups (Fig. 4a-c). Details of these pathways are provided in Fig. 4 and Additional file 3: Dataset S2. On the basis of the overlap analysis, sixteen pathways overlapped between PPC and AMG, 9 between PPC and HPC, and 13 between AMG and HPC (Fig. 4d). Thus, more metabolic pathways, including metabolism of amino acids, purine metabolism, and oxidation of fatty acids, overlapped between PPC and AMG than in the other comparisons. The sets of metabolites that differed between FC and control groups in all three brain regions exhibited enrichment in 8 pathways: arginine and proline metabolism, citric acid cycle, glutamate metabolism, glycine and serine metabolism, purine metabolism, pyruvate metabolism, tryptophan metabolism, and Warburg effect (Fig. 4d and Additional file 3: Dataset S2). Among 22 overlapping metabolic pathways on comparing each brain tissue with all other brain tissues, 17 pathways with high ranks in PPC were identified (Additional file 3: Dataset S2). Therefore, we performed pathway analysis that integrated metabolite set enrichment analysis and pathway topology analysis using the datasets of PPC metabolites and KEGG database in MetaboAnalyst software to identify the impact value of each pathway and the importance value of each metabolite (Additional file 4: Fig. S2). The pathways with high significance $(p$-value $<0.01)$ and impact value, and the metabolites with $p$-values $<0.1$ and importance value in these pathways are listed in Table 1.

In PPC, pantothenate and CoA biosynthesis had the highest significance and impact value. 4'-Phosphopantetheine had the highest significance and importance value in this pathway. Purine metabolism had a high significance level in pathway analysis. Various metabolites were significantly changed in purine metabolism, although each metabolite had a relatively low importance value. Glutathione metabolism had comparatively high significance and impact value. Glutathione had a high importance value in this pathway (Table 1). ADPribose (ADPR), a major metabolite in $\mathrm{NAD}^{+}$-dependent signaling, had the highest significance among all metabolites in PPC. Other metabolites with significance ( $p$-value $<0.05)$, such as ADP-ribose 2 -phosphate (ADPRP; $p$-value $<0.0001$ ), cyclic ADP-ribose (cADPR; $p$-value $=0.0275)$, and NADH $(p$-value $=0.0199)$ are also involved in $\mathrm{NAD}^{+}$-dependent signaling. Therefore, $\mathrm{NAD}^{+}$-dependent signaling seems to be one of the pathways perturbed by fear retrieval in PPC.

Analysis of significantly altered metabolites and pathways prompted us to depict representative pathways (Fig. 5). Pantothenate and CoA biosynthesis, purine metabolism, glutathione metabolism, and $\mathrm{NAD}^{+}$-dependent signaling were the pathways most perturbed in PPC by fear memory retrieval. Major metabolites of these pathways were significantly regulated in PPC with $p$-values $<0.05$ and fold change $\geq|1.2|$. Fear memory retrieval increased the level of $4^{\prime}$-phosphopantetheine, xanthine, and glutathione and decreased the level of ADP in PPC (Fig. 6a-d). In AMG, the level of xanthine tended to increase and ADP level decreased significantly, similar to PPC (Fig. 6b and c). In contrast, glutathione level was significantly reduced in HPC (Fig. 6d). In PPC, ADPR and ADPRP were increased, whereas CADPR and NADH were decreased by fear memory retrieval (Fig. 6e-h). Fear retrieval also increased the level of ADPR and ADPRP but decreased the level of cADPR in AMG (Fig. 6e-g). Notably, the trends of changes in NADH in both AMG and HPC were similar to that in PPC (Fig. 6h). Those metabolites were significantly regulated and could be used as biomarkers specific to conditioned fear memory in PPC.

\section{Discussion}

Our study revealed that fear memory retrieval significantly changed PPC, AMG, and HPC metabolites and perturbed related metabolic pathways. Conditioned fear memory is a risk factor for mental disorders such as anxiety, depression, and PTSD [36, 37]. Numerous studies have investigated metabolism underlying various stress-related psychiatric disorders by using models including conditioned fear memory in AMG and HPC. For example, in a rat model of chronic unpredictable mild stress (a commonly used model of depression), most differential metabolites in HPC were related to amino acid metabolism including that of glutamine and to lipid metabolism including that of cholesterol, 
a

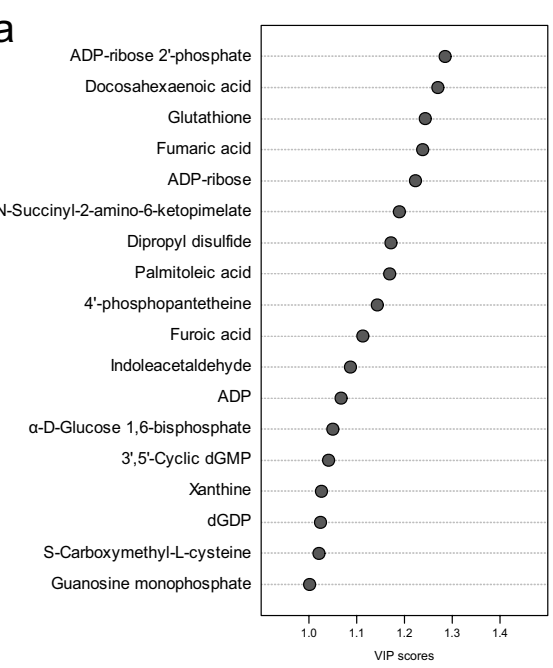

b

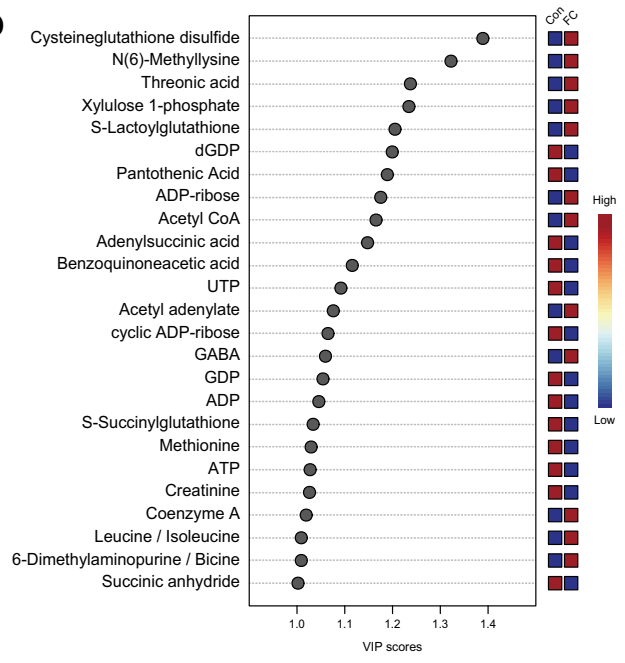

C

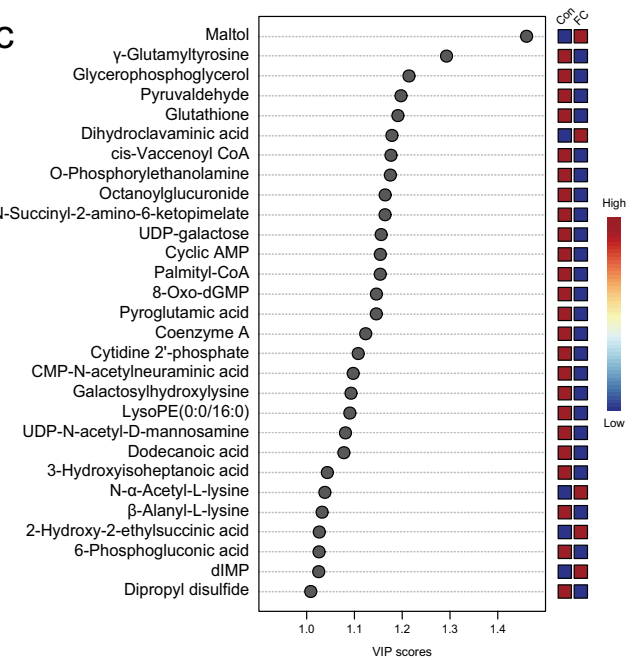

d

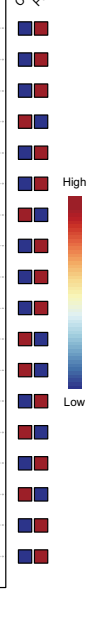

e

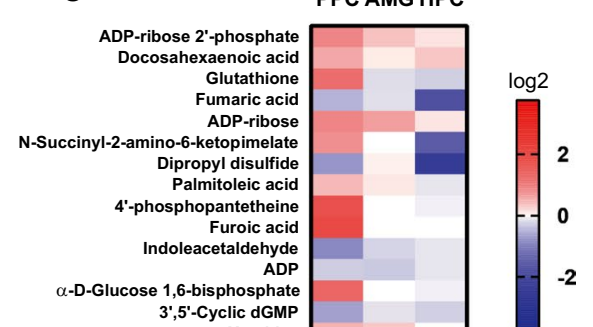

AMG HPC

Fig. 3 (See legend on previous page.) 


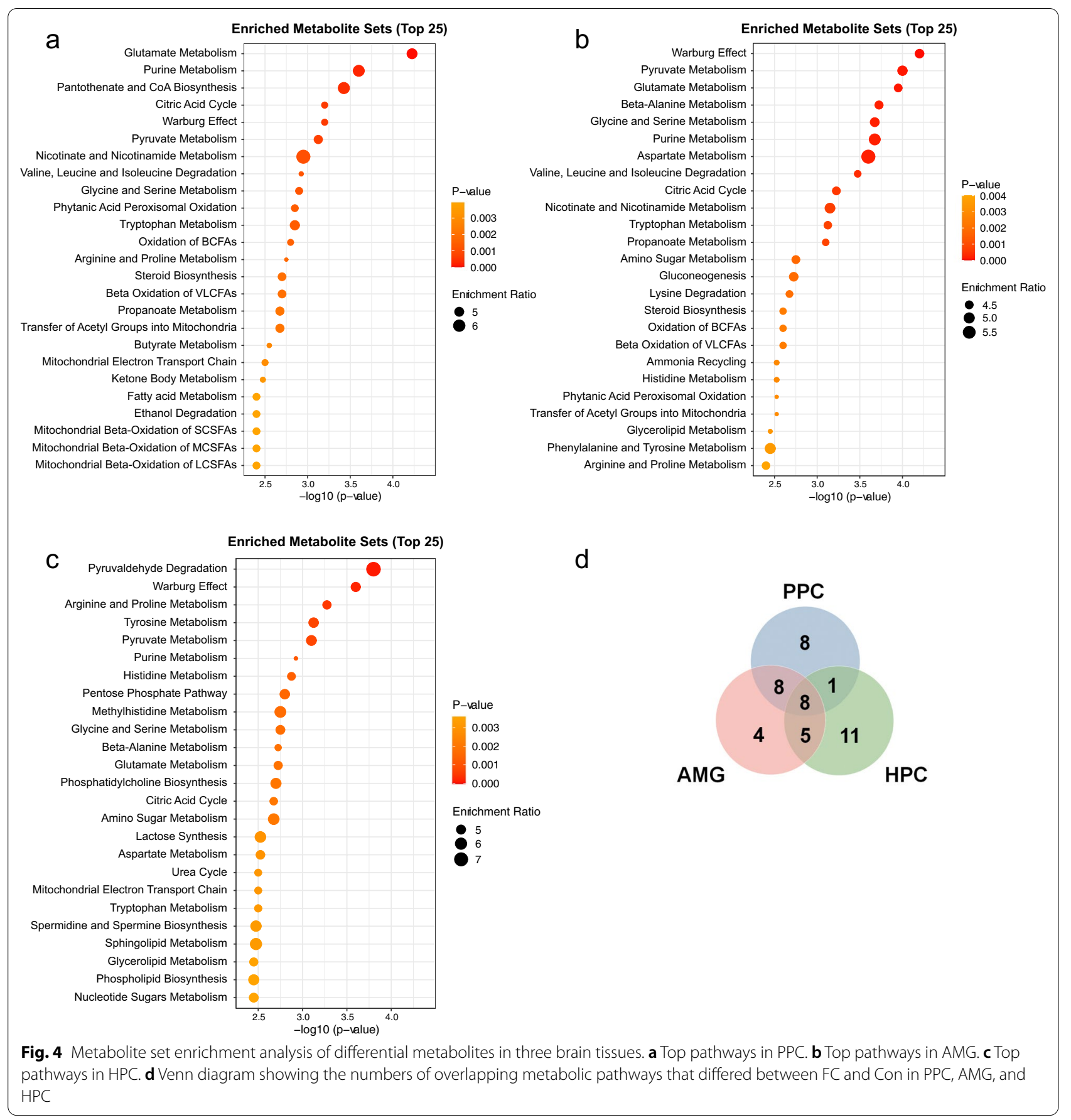

although some metabolites in glucose metabolism and the tricarboxylic acid cycle were also significantly changed [38]. In the HPC of mice exposed to a different chronic unpredictable mild stress condition, the levels of urea, phosphoric acid, glutamine, and cholesterol were increased and those of $\mathrm{N}$-carboxy-glycine, hexadecanoic acid, and octadecanoic acid were decreased [39]. Purine metabolism in HPC was perturbed in both chronic social defeat stress and fear conditioning mouse models $[35,40]$. In the HPC of susceptible mice that received chronic social defeat stress, the hyperfunction of the fatty acid beta-oxidation cycle was also shown [35]. Overall, various metabolic pathways including amino acid, lipid, carbohydrate, and purine metabolism are affected in HPC in diverse stress models. Other studies suggest that AMG metabolites 
Table 1 Pathway analysis of PPC metabolites altered in fear retrieval

\begin{tabular}{|c|c|c|c|c|c|c|c|}
\hline Pathway name & $p$-value & FDR & Impact & Metabolites & Importance & FC & $p$-value \\
\hline \multirow[t]{2}{*}{ Pantothenate and CoA biosynthesis ${ }^{\mathrm{a}}$} & 0.0003 & 0.007 & 0.4107 & PPanSH & 0.2357 & 3.18 & 0.0006 \\
\hline & & & & $\mathrm{COA}$ & 0.1750 & 1.31 & 0.0677 \\
\hline \multirow[t]{8}{*}{ Purine metabolism } & 0.0005 & 0.007 & 0.1366 & GMP & 0.0609 & 1.16 & 0.0186 \\
\hline & & & & Xanthine & 0.0297 & 1.36 & 0.0050 \\
\hline & & & & ADP & 0.0210 & 0.83 & 0.0114 \\
\hline & & & & dGDP & 0.0084 & 0.76 & 0.0398 \\
\hline & & & & ATP & 0.0070 & 0.79 & 0.0470 \\
\hline & & & & Deoxyguanosine & 0.0051 & 1.22 & 0.0156 \\
\hline & & & & Adenine & 0.0046 & 1.33 & 0.0316 \\
\hline & & & & ADPR & 0.0000 & 1.79 & $<0.0001$ \\
\hline \multirow[t]{2}{*}{ Tryptophan metabolism } & 0.0012 & 0.012 & 0.0139 & Indoleacetaldehyde & 0.0139 & 0.57 & 0.0036 \\
\hline & & & & Acetyl-CoA & 0.0000 & 1.16 & 0.0184 \\
\hline \multirow[t]{3}{*}{ Pyruvate metabolism } & 0.0017 & 0.013 & 0.1540 & Acetyl-CoA & 0.1540 & 1.16 & 0.0184 \\
\hline & & & & Acetyl adenylate & 0.0000 & 0.55 & 0.0145 \\
\hline & & & & Fumarate & 0.0000 & 0.72 & 0.0595 \\
\hline \multirow[t]{2}{*}{ Glutathione metabolism $^{\mathrm{a}}$} & 0.0066 & 0.029 & 0.2560 & Glutathione & 0.2560 & 2.22 & 0.0444 \\
\hline & & & & Acetyl-CoA & 0.0000 & 1.16 & 0.0184 \\
\hline \multirow[t]{3}{*}{ Citrate cycle (TCA cycle) } & 0.0081 & 0.029 & 0.0992 & Acetyl-CoA & 0.0367 & 1.16 & 0.0184 \\
\hline & & & & Succinate & 0.0327 & 1.14 & 0.0977 \\
\hline & & & & Fumarate & 0.0298 & 0.72 & 0.0595 \\
\hline
\end{tabular}

FC fold change, FDR false discovery rate, ADPR ADP-ribose, PPanSH 4'-Phosphopantetheine

${ }^{\text {a }}$ Pathway with high impact score $(>0.2)$

are also altered in several stress conditions associated with developing mental disorders. Perturbation in amino acid, fatty acid, and carbohydrate metabolism was observed in the AMG in the chronic unpredictable mild stress rat model, similar to the findings in HPC [41]. The level of the amino acid serine was significantly decreased in the AMG of dominant hamsters following a social defeat encounter [34]. Our study showed that various metabolites in amino acid, carbohydrate, and purine metabolism were changed in the AMG and HPC in the conditioned fear model. Especially, lipid metabolisms including phosphatidylcholine biosynthesis and sphingolipid metabolism were significantly regulated in the HPC.

Some studies have investigated the metabolites and pathways that are differentially regulated in the PFC and nucleus accumbens as well as AMG and HPC in social defeat stress and chronic restraint stress models of mental disorders [34, 42]. Although the PPC is implicated in fear learning and memory $[9,29]$, the metabolites and metabolic pathways that are affected in PPC remain unknown. In our study, UPLC-MS/MS based metabolomics analysis data showed that purine metabolism, pantothenate and CoA biosynthesis, glutathione metabolism, and $\mathrm{NAD}^{+}$-dependent signaling were the most perturbed pathways in the retrieval of conditioned fear memory.

Our study suggests that pantothenate and CoA biosynthesis, which had the highest significance and impact value in pathway analysis, was the most perturbed pathway in PPC by conditioned fear memory. In pantothenate and CoA biosynthesis, pantothenate is phosphorylated by pantothenate kinase and converted to 4'-phosphopantetheine, an intermediate of this pathway [43]. Pantothenate kinase KO mice displayed increases in gene expressions of dopamine D1 receptor (D1R) and dopamine D2 receptor (D2R) in globus pallidus-containing region including striatum [44]. Interestingly, local infusion of D1R antagonist SCH23390 into AMG, HPC or PFC prior to the fear conditioning impairs fear responses such as freezing $[45,46]$. Dentate gyrus-specific D1R KO mice showed significant fear memory deficits [47]. To test the change in gene expression of D1r in PPC, we measured and compared the mRNA level of D1r in PPC of the control and FC groups. The mRNA level of D1r was significantly increased in the FC group (Additional file 5: Fig. S3) whereas those of other proteins such as tyrosine hydrolase and D2R related to dopamine metabolism were barely detected in PPC (data not shown). It is possible that the perturbed pantothenate and CoA biosynthesis 


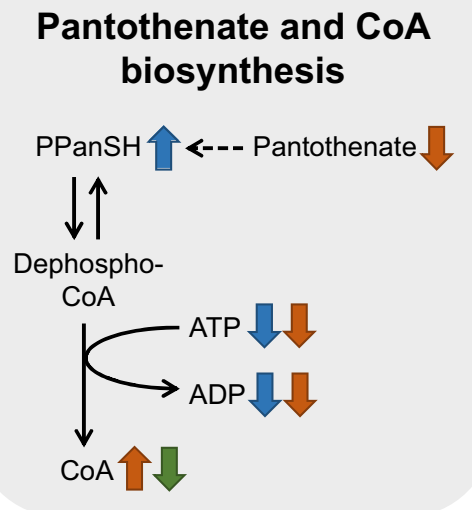

\section{Glutathione metabolism}

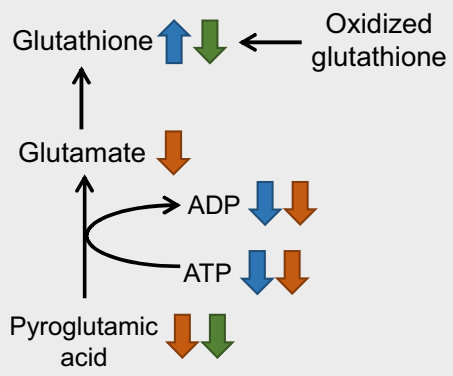

Purine metabolism

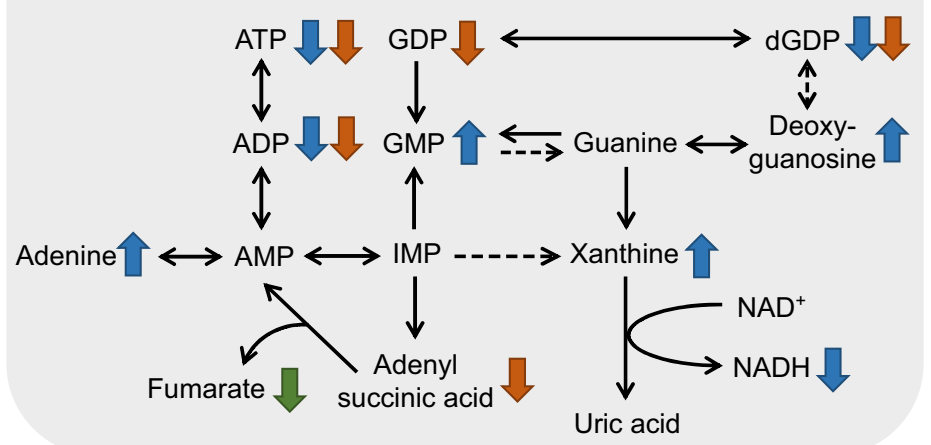

$\mathrm{NAD}^{+}$dependent signaling

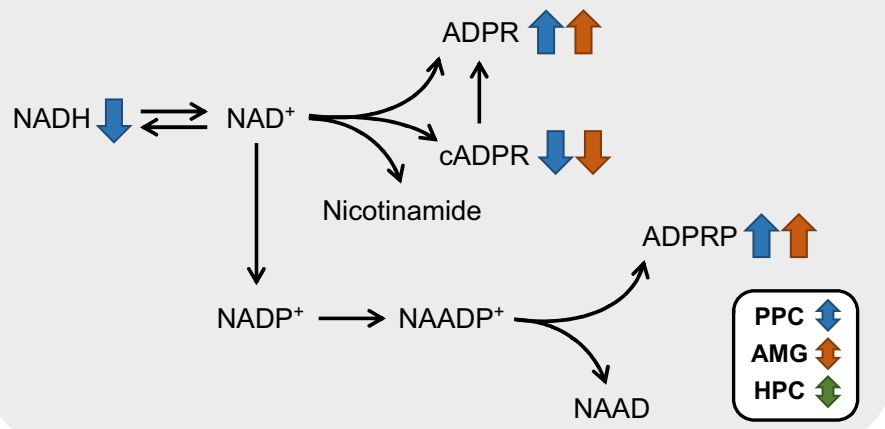

Fig. 5 The perturbed major metabolic pathways of PPC and significantly different metabolites in the fear retrieval condition. Blue arrows, significantly changed metabolites of PPC; red arrows, significantly changed metabolites of AMG; green arrows, significantly changed metabolites of HPC. PPanSH 4'-Phosphopantetheine, ADPR ADP-ribose, ADPRP ADP-ribose 2'-phosphate, CADPR cyclic ADP-ribose, NAADP' nicotinic acid adenine dinucleotide phosphate, NAAD nicotinic acid adenine dinucleotide

pathway upregulates D1r expression in PPC and the increase in $D 1 r$ might be involved in fear responses as in the AMG, HPC or PFC.

In our data, purine metabolism included most metabolites that were changed by fear retrieval in PPC. Xanthine might be important among the significantly altered metabolites in this pathway. Xanthine is formed by the oxidation of hypoxanthine, and the intra-striatal administration of hypoxanthine impairs fear memory learning, consolidation, and retrieval [48]. One paper showed that electronic foot shock increased serum Xanthine [49]. Xanthine was significantly increased in PPC but was not detected in HPC. In AMG, the trend of changes in the level of xanthine was similar to that in PPC. Additionally, ADP level was significantly decreased in PPC and AMG. Since ADP is related to various metabolic pathways, further studies of its functions and related pathways in the regulation of fear memory in PPC are needed.
In glutathione metabolism, we found that the level of glutathione was increased in PPC and decreased in HPC. Glutathione is an anti-oxidant, and an increase in glutathione levels plays a role in protection from oxidative stress [50]. The level of oxidative stress is reportedly elevated in a PTSD model [51]. One study suggested that the level of cystine, which is converted to cysteine, a precursor to glutathione, increases under social defeat stress [34]. The sensitivity to oxidative stress varies in different brain regions. It was suggested that HPC may be a sensitive target of toxic oxidative bursts compared to other brain regions in Carioca high-conditioned freezing animals which present high defensive freezing responses to contextual fear [52]. Therefore, glutathione may be rapidly oxidized by oxidizing agents. As such, our study shows that glutathione levels seem to be decreased by conditioned fear-induced oxidative stress in HPC. In contrast, the increased glutathione levels of PPC might be due to a protective mechanism against oxidative 

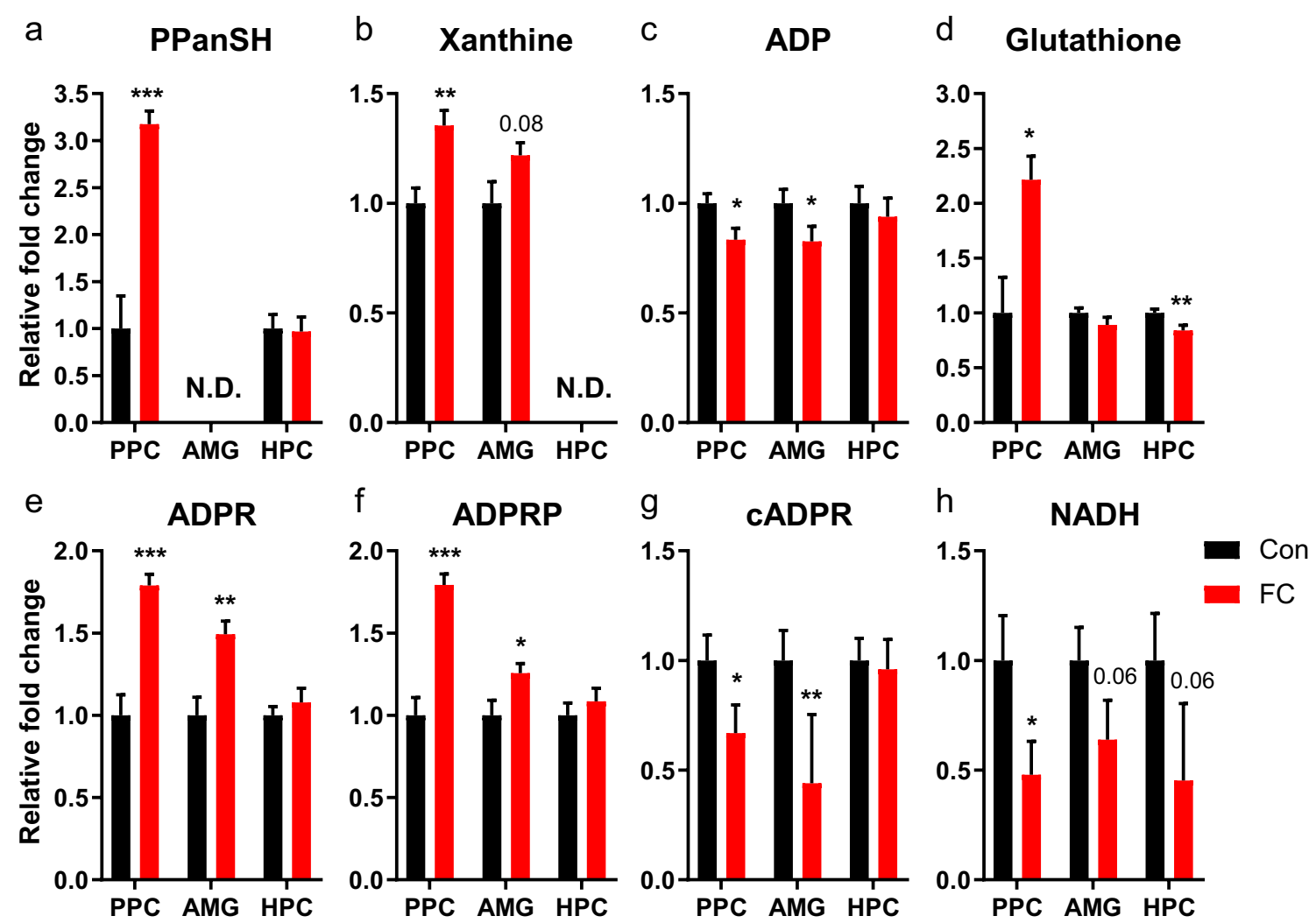

Fig. 6 Changes of representative metabolites in the most perturbed metabolic pathways. Each metabolite was presented with $p$-value $<0.05$, fold change $\geq|1.2|$, and high importance on each pathway in PPC. The fold change and statistical significance of each metabolite for FC vs. Con in PPC, AMG, and HPC were presented (Con-PPC, $n=16$ mice; FC-PPC, $n=17$ mice; Con-AMG, $n=16$ mice; FC-AMG, $n=17$ mice; Con-HPC, $\mathrm{n}=16$ mice; FC-HPC, $\mathrm{n}=13$ mice). Two-tailed unpaired $t$-test, data are mean $\pm \mathrm{SEM} ;{ }^{*} p<0.05,{ }^{* *} p<0.01,{ }^{* * *} p<0.001$. N.D. not determined. PPanSH 4'-Phosphopantetheine, ADPR ADP-ribose, ADPRP ADP-ribose 2'-phosphate, CADPR cyclic ADP-ribose

stress, suggesting that PPC may be less sensitive to oxidative stress compared to HPC.

Several studies have demonstrated that various enzymes including CD38, CD157, and SARM1 produce both cADPR and ADPR using $\mathrm{NAD}^{+}$as substrate [5356]. CD38 also hydrolyzes CADPR to ADPR and nicotinic acid adenine dinucleotide phosphate to ADPRP [56, 57]. These enzymes are involved in fear conditioning and fear memory retrieval. CD38 is important in fear learning processes including fear acquisition, extinction, and retrieval $[58,59]$. CD157 is expressed in the AMG and regulates anxiety-related and depression-like behaviors including fear response [60]. SARM1 is also expressed in the AMG and has roles in both HPC- and AMG-dependent fear conditioning [61]. Thus, our results suggest that changes in metabolites such as ADPR, cADPR, ADPRP, and $\mathrm{NADH}$ are associated with $\mathrm{NAD}^{+}$-related metabolism and the perturbed metabolic pathway in PPC and AMG might be involved in the conditioned fear memory.

Future comprehensive metabolomics analyses using various models such as fear extinction and fear renewal may pave the way toward better understanding of the significance of metabolites and metabolic pathways in fear memory regulation including destabilization, reconsolidation, and extinction after fear memory retrieval, thereby providing ideas for therapeutic strategies for the treatment of fear-related mental disorders.

\section{Conclusion}

We analyzed and compared changes of metabolites in the HPC, AMG, and PPC in conditioned fear memory. One of the purposes of this study was to identify the metabolites that could be exploited for the development of potential biomarkers for fear-related mental illnesses. While our analysis of fear memory-induced changes in metabolites within selected brain regions indicated that many small molecules are changed significantly between FC and control mice, our study identified 4'-phosphopantetheine, xanthine, glutathione, ADPR, cADPR, and ADPRP as putative biomarkers and suggests that the corresponding metabolic pathways are related to fear memory in PPC. 


\section{Methods}

\section{Animals}

Male C57BL/6 mice (8 weeks old; purchased from The Koatech) were maintained in a controlled-light environment (12 h light and $12 \mathrm{~h}$ dark cycle; lights on from 7 am to $7 \mathrm{pm}$ ) with ad libitum access to standard laboratory chow (Teklad 2018) and water. Mice were handled daily for 1 week prior to behavioral procedures for fear conditioning and retrieval tests. All behavioral procedures were performed in accordance with the guidelines on care and use of laboratory animals as approved by the DGIST Institutional Animal Care and Use Committee to minimize animal suffering.

\section{Fear conditioning procedure}

The fear conditioning procedure was performed in accordance with a previous protocol [9]. For the retrieval test, the context and day conditions of the procedure were modified because fear extinction training was not required.

The mouse test cage $(17.78 \times 17.78 \times 30.48 \mathrm{~cm}$; Coulbourn Instruments) used for fear conditioning consisted of gray walls and a stainless-steel shock grid floor. The grid floor was composed of bars that allowed the delivery of electric foot shocks. Before fear conditioning and the retrieval test, the wall and floor were cleaned with $75 \%$ ethanol.

On the day of fear conditioning, mice in the FC group were acclimated in the test cage for $300 \mathrm{~s}$, and a tone (CS; $2.8 \mathrm{kHz}, 85 \mathrm{~dB}, 30 \mathrm{~s}$ ) was then co-terminated with an aversive foot shock (US; $0.2 \mathrm{~mA}, 0.5 \mathrm{~s}$ ), which was presented 6 times at 300, 390, 500, 630, 740, and $870 \mathrm{~s}$ [62]. The variable time interval between trials which are tones co-terminated with a foot shock was used to minimize the possibility of the animals expecting the next trial. The retrieval test was performed on the next day. After exploration for $300 \mathrm{~s}$, the same tone, without an electric foot shock, was played twice, at 300 and 390 s. The mice in the control group were exposed to the same context and tones at the same times as in the FC group without receiving foot shocks. Freezing is defined as the suppression of movement with the exception of respiration. FreezeFrame software (Coulbourn Instruments) was used to score freezing behavior, defined as mouse immobility lasting more than $1 \mathrm{~s}$ in accordance with a reference [9].

\section{Tissue collection}

After behavioral procedures, the mice were anesthetized with isoflurane, and brains were collected. Brains were sliced coronally at $1 \mathrm{~mm}$ thickness by using a brain matrix. The PPC and AMG punches $(2.0 \mathrm{~mm}$ diameter for PPC and $1.5 \mathrm{~mm}$ diameter for AMG) were collected bilaterally from a slice section $(1.0 \mathrm{~mm}$; from -1.0 to $-2.0 \mathrm{~mm}$ relative to bregma). The HPC tissues were manually dissected and cut bilaterally in two slice section $(1.0 \mathrm{~mm}$ each; from -1.0 to $-3.0 \mathrm{~mm}$ relative to bregma). The tissues were flash frozen in liquid nitrogen and stored at $-80{ }^{\circ} \mathrm{C}$ until metabolite extraction. In some brain regions, tissue was not assayed because of inaccurate dissections.

\section{Sample preparation}

PPC and AMG tissues were extracted with $500 \mu \mathrm{l}$ of Chilled extraction solvent (acetonitrile:methanol:water, 2:2:1, v/v) containing $5 \mu \mathrm{M} \mathrm{ATP}-{ }^{13} \mathrm{C}_{10}$ (Sigma-Aldrich), which was used as an internal standard. HPC tissue was extracted with $800 \mu \mathrm{l}$ of the same extraction solvent with the internal standard. The mixtures were sonicated for about $7 \mathrm{~min}$ (10 cycles), vortexed for $10 \mathrm{~min}$, and then incubated at $-20{ }^{\circ} \mathrm{C}$ for $1 \mathrm{~h}$. The samples were centrifuged at $18,000 \times g$ for $10 \mathrm{~min}$ at $4{ }^{\circ} \mathrm{C}$. The supernatants were collected and stored at $-20{ }^{\circ} \mathrm{C}$. The pellets were re-extracted using $200 \mu \mathrm{l}$ aliquots of the extraction solvent and the procedure was repeated. The supernatants were combined and dried under nitrogen flow in a nitrogen evaporator. The dry extracts were reconstituted with $100 \mu \mathrm{l}$ of $60 \%$ acetonitrile (ACN) and filtered through a $0.22 \mu \mathrm{m}$ PTFE filter before analysis.

\section{UPLC-MS analysis for data acquisition}

All samples were analyzed using an Agilent 1290 UPLC system coupled to an Agilent 6530 Q-TOF MS (Agilent Technologies) in positive or negative ESI mode. The instrument parameters were as follows: gas temperature $325{ }^{\circ} \mathrm{C}$, drying gas $11 \mathrm{l} / \mathrm{min}$, nebulizer $30 \mathrm{psig}$, fragment voltage $170 \mathrm{~V}$, skimmer $60 \mathrm{~V}$, and capillary voltage $\pm 3,500 \mathrm{~V}$ (nozzle voltage $500 \mathrm{~V}$; only negative mode). Internal mass calibration was performed using reference masses during the runs.

The sample $(10 \mu \mathrm{l})$ was injected into a Merck ZICcHILIC column $(100 \times 2.1 \mathrm{~mm}, 3 \mu \mathrm{m})$ heated to $40{ }^{\circ} \mathrm{C}$ in a column oven. The mobile phase A consisted of $9 / 1(\mathrm{v} / \mathrm{v})$ water/ACN containing $10 \mathrm{mM}$ ammonium acetate $(\mathrm{pH}$ 6.9) and the mobile phase B consisted of $1 / 9(\mathrm{v} / \mathrm{v})$ water/ ACN containing $10 \mathrm{mM}$ ammonium acetate and $0.1 \%$ acetic acid ( $\mathrm{pH} 7.3)$. Flow rate was $0.3 \mathrm{ml} / \mathrm{min}$ and the gradient program was as follows: $0-20 \mathrm{~min}, 87.5-37.5 \%$ B; 20-21 min, 37.5\%; column wash: $21-21.5 \mathrm{~min}, 37.5-$ $0 \% \mathrm{~B} ; 21.5-23.5 \mathrm{~min}, 0 \% \mathrm{~B} ; 23.5-24 \mathrm{~min} 0-87.5 \% \mathrm{~B}$; the initial condition $(87.5 \% \mathrm{~B})$ was maintained for $7 \mathrm{~min}$ for equilibration. Untargeted data acquisition on the UPLCMS was performed using Agilent MassHunter Workstation Data Acquisition software (Agilent Technologies). 


\section{Data filtering and visualization}

The UPLC-MS data were deconvoluted into individual chemical peaks and used to find a "feature" that describes a chemical entity in a chromatogram via the algorithms "Molecular Feature Extractor" and "Find by Formula" in Agilent MassHunter Qualitative Analysis B.07.00 software (Agilent Technologies). The results were used for subsequent statistical analysis and data visualization in Mass Profiler Professional (MPP) software (Agilent Technologies). To create feature lists, the data were filtered on the basis of the number of entities detected in at least one condition (FC or control) in each brain region. Features appearing in more than half of the samples in at least one group were used for subsequent analyses.

PLS-DA was performed to display the variance in samples within the groups. To generate the PLS-DA data, the MetaboAnalyst web tool (www.metaboanalyst.ca) was used. All data were normalized by using an auto-scaling method that attempts to scale and center the data by dividing each value by standard deviation. By extracting the PLS-DA score values, the visualization aid was generated with a $95 \%$ confidence interval. VIP score plots were also generated from the PLS-DA function of MetaboAnalyst. After PLS-DA, the fold change data of metabolites with high VIP scores were log 2 transformed and then displayed as a heat map in GraphPad Prism 8 software. For visual comparison, the concentration changes in the heat map were shown using a color scale ranging from +3 (red) to -3 (blue).

\section{UPLC-MS/MS analysis for identification of features}

The metabolic features that differed between FC and control were identified by accurate mass and isotopic abundance pattern matching to the METLIN database in MPP. To confirm the identity of these metabolites, UPLC-MS/MS analysis was subsequently performed. The lists of compounds created in MPP, which included $\mathrm{m} / \mathrm{z}$ and retention time values, were used as targets in MS/ MS analysis. Several brain samples were re-analyzed by targeted MS/MS analysis on the Q-TOF MS. The sample sizes of PPC, AMG, and HPC for UPLC-MS/MS are 25,26 , and 29 , respectively. MS/MS spectra were generated at a given collision energy and the sample spectra were matched to a MS/MS spectral library of standards in METLIN database by using Agilent MassHunter Molecular Structure Correlator B.05.00 software (Agilent Technologies).

\section{Pathway analysis}

Metabolite set enrichment analyses in the three brain regions were performed using MetaboAnalyst. The analyses were based on SMPDB libraries containing 99 groups of metabolite sets that are linked to KEGG database and HMDB. To determine the significance of changes in metabolites and related pathways revealed by enrichment analysis, pathway analysis of PPC was performed using KEGG database in MetaboAnalyst. The pathway analysis module combines results from enrichment analysis and pathway topology analysis.

\section{Real-time quantitative PCR}

Total RNA from brain tissue was isolated with TRIzol reagent (Invitrogen) according to the manufacturer's instructions. The concentration of RNA was determined using a NanoDrop spectrophotometer (Thermo Scientific). cDNA was synthesized from $1.6 \mu \mathrm{g}$ RNA using the GoScript $^{\mathrm{TM}}$ Reverse Transcription System (Promega) as described previously [63]. In a CFX 96 Real-Time system (Bio-Rad), TB Green (TaKaRa Biotechnology) was used for quantitative PCR estimation of the expression of $D 1 r$ and Gapdh genes. The following primer pairs were used: $D 1 r$, forward primer, 5'-CAGCCTTCATCCTGA TTAGCGTAG-3' ${ }^{\prime}$, reverse primer, $5^{\prime}$-CTTATGAGGGAG GATGAAATGGCG-3'. Gapdh, forward primer, 5'-ATC ACTGCCACCCAGAAGAC-3', reverse primer, 5'-ACA CATTGGGGGTAGGAACA-3'. The expression level was normalized to that of Gapdh as an endogenous control.

\section{Statistical analysis}

Data were analyzed using univariate and multivariate analyses, including unpaired $t$-test for independent pairs of groups. The effects of fear retrieval on classification of the samples of each brain tissue were compared. A cut-off value of asymptotic $p<0.05$ and threshold $>1.1$ were considered significant in unpaired $t$-test using MPP software. The pathway analyses were performed and the $p$-value and impact value were assessed using MetaboAnalyst software. All data are presented as mean \pm standard error of the mean (SEM). For comparisons of data between two groups, the two-tailed unpaired $t$-test was used. For comparing freezing percentage data of more than three groups, one-way ANOVA followed by Tukey's multiple comparisons or two-way repeated-measures ANOVA was performed using GraphPad Prism 8 Software. A $p$-value of $<0.05$ was considered statistically significant.

\section{Abbreviations}

ACN: Acetonitrile; ADPR: ADP-ribose; ADPRP: ADP-ribose 2'-phosphate; AMG: Amygdala; CADPR: Cyclic ADP-ribose; CS: Conditioned stimulus; D1R: Dopamine D1 receptor; D2R: Dopamine D2 receptor; FC: Fear conditioning; FDR: False discovery rate; HPC: Hippocampus; MPP: Mass profiler professional; PFC: Prefrontal cortex; PLS-DA: Partial least squares discriminant analysis; PPC: Posterior parietal cortex; PTSD: Post-traumatic stress disorder; UPLC-MS: Ultra-performance liquid chromatography-mass spectrometry; UPLC-MS/MS: Ultra-performance liquid chromatography-tandem mass spectrometry; US: Unconditioned stimulus; VIP: Variable importance in projection. 


\section{Supplementary Information}

The online version contains supplementary material available at https://doi. org/10.1186/s13041-021-00863-x.

Additional file 1: Fig. S1. The 3D score plots of PLS-DA in three brain tissues. a PPC. b AMG. $\mathbf{c}$ HPC (Con-PPC, $n=16$ mice; FC-PPC, $n=17$ mice; Con-AMG, $n=16$ mice; FC-AMG, $n=17$ mice; Con-HPC, $n=16$ mice; $F C$ $\mathrm{HPC}, \mathrm{n}=13$ mice)

Additional file 2: Dataset S1. Details of statistical analysis and fold changes of overlapping metabolites in Fig. 3d and e. Significant increases in fold change value are highlighted in red and significant decreases are highlighted in blue. FC, fold change; N.D., not determined.

Additional file 3: Dataset S2. Details of enrichment analysis in three brain regions in Fig. 4. Gray boxes, metabolic pathways overlapping between all three brain regions; pink boxes, metabolic pathways overlapping between PPC and AMG; orange boxes, metabolic pathways overlapping between AMG and HPC; green boxes, metabolic pathways overlapping between PPC and HPC; white boxes, metabolic pathways identified in a single brain region. FDR, false discovery rate.

Additional file 4: Fig. S2. Pathway analysis of metabolites changed by fear retrieval in PPC.

Additional file 5: Fig. S3. The relative mRNA level of the dopamine D1 receptor in PPC (Con, $\mathrm{n}=6$ mice; FC, $\mathrm{n}=6$ mice). Two-tailed unpaired $t$-test, data are mean $\pm S E M ;{ }^{*} p<0.05$.

\section{Acknowledgements}

Not applicable.

\section{Authors' contributions}

YJ and EKK conceived and designed the study, interpreted the results, and wrote the manuscript. YJ and YL planned and performed the experiments and analyzed the data. JY supported the experiments and analysis of the data. All authors read and approved the final manuscript.

\section{Funding}

This research was supported by the National Research Foundation of Korea under grant numbers 2018M3C7A1056275 and 2020R1A2B5B02001468.

\section{Availability of data and materials}

All data generated or analyzed during this study are included in this published article.

\section{Declarations}

\section{Ethics approval and consent to participate}

All experimental procedures were in accordance with the DGIST guidelines and were approved by the DGIST Institutional Animal Care and Use Committee (DGIST-IACUC-19011702-0000).

\section{Consent for publication}

Not applicable.

\section{Competing interests}

The authors declare that they have no competing interests.

\section{Author details}

${ }^{1}$ Department of Brain and Cognitive Sciences, Daegu Gyeongbuk Institute of Science and Technology (DGIST), Daegu 42988, Republic of Korea. ${ }^{2} \mathrm{Neu}$ rometabolomics Research Center, Daegu Gyeongbuk Institute of Science and Technology (DGIST), Daegu 42988, Republic of Korea.

Received: 25 May 2021 Accepted: 23 September 2021 Published online: 06 October 2021

\section{References}

1. Uher R, Zwicker A. Etiology in psychiatry: embracing the reality of poly-gene-environmental causation of mental illness. World Psychiatry. 2017; 16(2):121-9.

2. Garakani A, Murrough JW, Freire RC, Thom RP, Larkin K, Buono FD, et al. Pharmacotherapy of anxiety disorders: current and emerging treatment options. Focus. 2021;19(2):222-42.

3. Coleman JR, Peyrot WJ, Purves KL, Davis KA, Rayner C, Choi SW, et al. Genome-wide gene-environment analyses of major depressive disorder and reported lifetime traumatic experiences in UK Biobank. Mol Psychiatry. 2020;25(7):1430-46.

4. Sareen J. Posttraumatic stress disorder in adults: impact, comorbidity, risk factors, and treatment. Can J Psychiatry. 2014;59(9):460-7.

5. Bouton ME. Context, time, and memory retrieval in the interference paradigms of Pavlovian learning. Psychol Bull. 1993;114(1):80.

6. Bouton ME. Context, ambiguity, and classical conditioning. Curr Dir Psychol Sci. 1994;3(2):49-53.

7. Jones SV, Heldt SA, Davis M, Ressler KJ. Olfactory-mediated fear conditioning in mice: simultaneous measurements of fear-potentiated startle and freezing. Behav Neurosci. 2005:119(1):329.

8. Fadok JP, Darvas M, Dickerson TM, Palmiter RD. Long-term memory for pavlovian fear conditioning requires dopamine in the nucleus accumbens and basolateral amygdala. PLoS ONE. 2010;5(9):e12751.

9. Joo B, Koo JW, Lee S. Posterior parietal cortex mediates fear renewal in a novel context. Mol Brain. 2020;13(1):1-11.

10. Kim JJ, Jung MW. Neural circuits and mechanisms involved in Pavlovian fear conditioning: a critical review. Neurosci Biobehav Rev. 2006;30(2):188-202

11. Kim JJ, Fanselow MS. Modality-specific retrograde amnesia of fear. Science. 1992;256(5057):675-7.

12. Phillips R, LeDoux J. Differential contribution of amygdala and hippocampus to cued and contextual fear conditioning. Behav Neurosci. 1992;106(2):274.

13. Rudy JW, O'Reilly RC. Contextual fear conditioning, conjunctive representations, pattern completion, and the hippocampus. Behav Neurosci. 1999;113(5):867.

14. Dębiec J, Díaz-Mataix L, Bush DE, Doyère V, LeDoux JE. The amygdala encodes specific sensory features of an aversive reinforcer. Nat Neurosci. 2010;13(5):536

15. Knapska E, Macias M, Mikosz M, Nowak A, Owczarek D, Wawrzyniak M, et al. Functional anatomy of neural circuits regulating fear and extinction. Proc Natl Acad Sci. 2012;109(42):17093-8.

16. Sotres-Bayon F, Sierra-Mercado D, Pardilla-Delgado E, Quirk GJ. Gating of fear in prelimbic cortex by hippocampal and amygdala inputs. Neuron. 2012;76(4):804-12.

17. Orsini CA, Kim JH, Knapska E, Maren S. Hippocampal and prefrontal projections to the basal amygdala mediate contextual regulation of fear after extinction. J Neurosci. 2011;31(47):17269-77.

18. Jimenez JC, Berry JE, Lim SC, Ong SK, Kheirbek MA, Hen R. Contextual fear memory retrieval by correlated ensembles of ventral CA1 neurons. Nat Commun. 2020;11(1):1-11.

19. Matos MR, Visser E, Kramvis I, van der Loo RJ, Gebuis T, Zalm R, et al. Memory strength gates the involvement of a CREB-dependent cortical fear engram in remote memory. Nat Commun. 2019;10(1):1-11.

20. Andersen RA, Buneo CA. Intentional maps in posterior parietal cortex. Annu Rev Neurosci. 2002;25(1):189-220.

21. Morcos AS, Harvey CD. History-dependent variability in population dynamics during evidence accumulation in cortex. Nat Neurosci. 2016;19(12):1672-81.

22. Raposo D, Kaufman MT, Churchland AK. A category-free neural population supports evolving demands during decision-making. Nat Neurosci. 2014;17(12):1784

23. Snyder LH, Batista A, Andersen RA. Coding of intention in the posterior parietal cortex. Nature. 1997:386(6621):167-70.

24. Najafi F, Churchland AK. Perceptual decision-making: a field in the midst of a transformation. Neuron. 2018:100(2):453-62.

25. Andersen RA, Snyder LH, Bradley DC, Xing J. Multimodal representation of space in the posterior parietal cortex and its use in planning movements. Annu Rev Neurosci. 1997;20(1):303-30. 
26. Song Y-H, Kim J-H, Jeong H-W, Choi I, Jeong D, Kim K, et al. A neural circuit for auditory dominance over visual perception. Neuron. 2017:93(4):940-54.

27. Freedman DJ, Ibos G. An integrative framework for sensory, motor, and cognitive functions of the posterior parietal cortex. Neuron. 2018;97(6):1219-34.

28. Xu Y. The posterior parietal cortex in adaptive visual processing. Trends Neurosci. 2018;41(11):806-22.

29. Tayler KK, Tanaka KZ, Reijmers LG, Wiltgen BJ. Reactivation of neural ensembles during the retrieval of recent and remote memory. Curr Biol. 2013;23(2):99-106.

30. Griffiths W, Karu K, Hornshaw M, Woffendin G, Wang Y. Metabolomics and metabolite profiling: past heroes and future developments. Eur J Mass Spectrom. 2007;13(1):45-50.

31. Oldiges M, Lütz S, Pflug S, Schroer K, Stein N, Wiendahl C. Metabolomics: current state and evolving methodologies and tools. Appl Microbiol Biotechnol. 2007;76(3):495-511.

32. Kaddurah-Daouk R, Kristal BS, Weinshilboum RM. Metabolomics: a global biochemical approach to drug response and disease. Annu Rev Pharmacol Toxicol. 2008;48(1):653-83.

33. Kao C-Y, Anderzhanova E, Asara JM, Wotjak CT, Turck CW. NextGen brain microdialysis: applying modern metabolomics technology to the analysis of extracellular fluid in the central nervous system. Mol Neuropsychiatry. 2015;1(1):60-7.

34. Dulka BN, Bourdon AK, Clinard CT, Muvvala MB, Campagna SR, Cooper MA. Metabolomics reveals distinct neurochemical profiles associated with stress resilience. Neurobiol Stress. 2017:7:103-12.

35. Hamilton PJ, Chen EY, Tolstikov V, Peña CJ, Picone JA, Shah P, et al. Chronic stress and antidepressant treatment alter purine metabolism and beta oxidation within mouse brain and serum. Sci Rep. 2020;10(1):1-14.

36. Li X. Using the conditioned fear stress (CFS) animal model to understand the neurobiological mechanisms and pharmacological treatment of anxiety. Shanghai Arch Psychiatry. 2012.

37. Amstadter AB, Nugent NR, Koenen KC. Genetics of PTSD: fear conditioning as a model for future research. Psychiatric Ann. 2009;39(6).

38. Zhang Y, Yuan S, Pu J, Yang L, Zhou X, Liu L, et al. Integrated metabolomics and proteomics analysis of hippocampus in a rat model of depres sion. Neuroscience. 2018;371:207-20.

39. Zhao J, Jung Y-H, Jang C-G, Chun K-H, Kwon SW, Lee J. Metabolomic identification of biochemical changes induced by fluoxetine and imipramine in a chronic mild stress mouse model of depression. Sci Rep. 2015;5(1):1-8.

40. Koyanagi I, Sonomura K, Naoi T, Ohnishi T, Kaneko N, Sawamoto K, et al. Metabolic fingerprints of fear memory consolidation during sleep. Mol Brain. 2021;14(1):1-14.

41. Li X, Zhou X, Teng T, Fan L, Liu X, Xiang Y, et al. Multi-omics analysis of the amygdala in a rat chronic unpredictable mild stress model of depression. Neuroscience. 2021.

42. Liu L, Zhou X, Zhang Y, Liu Y, Yang L, Pu J, et al. The identification of metabolic disturbances in the prefrontal cortex of the chronic restraint stress rat model of depression. Behav Brain Res. 2016;305:148-56.

43. Shimizu S, Kubo K, Tani Y, Ogata K. Purification and properties of pantothenate kinase from Brevibacterium ammoniagenes IFO 12071. Agric Biol Chem. 1973;37(12):2863-70.

44. Jeong SY, Hogarth P, Placzek A, Gregory AM, Fox R, Zhen D, et al. 4'-Phosphopantetheine corrects CoA, iron, and dopamine metabolic defects in mammalian models of PKAN. EMBO Mol Med. 2019;11(12):e10489.

45. Heath FC, Jurkus R, Bast T, Pezze MA, Lee JL, Voigt JP, et al. Dopamine D1-like receptor signalling in the hippocampus and amygdala modulates the acquisition of contextual fear conditioning. Psychopharmacology. 2015;232(14):2619-29.

46. Stubbendorff C, Hale E, Cassaday HJ, Bast T, Stevenson CW. Dopamine D1-like receptors in the dorsomedial prefrontal cortex regulate contextual fear conditioning. Psychopharmacology. 2019;236(6):1771-82.
47. Sariñana J, Kitamura T, Künzler P, Sultzman L, Tonegawa S. Differential roles of the dopamine 1-class receptors, D1R and D5R, in hippocampal dependent memory. Proc Natl Acad Sci. 2014;111(22):8245-50.

48. Bavaresco CS, Ben J, Chiarani F, Netto CA, de Souza Wyse AT. Intrastriatal injection of hypoxanthine impairs memory formation of stepdown inhibitory avoidance task in rats. Pharmacol Biochem Behav. 2008;90(4):594-7.

49. Fan K-Q, Li Y-Y, Wang H-L, Mao X-T, Guo J-X, Wang F, et al. Stress-induced metabolic disorder in peripheral CD4+ T cells leads to anxiety-like behavior. Cell. 2019;179(4):864-79.

50. Shih AY, Erb H, Sun X, Toda S, Kalivas PW, Murphy TH. Cystine/glutamate exchange modulates glutathione supply for neuroprotection from oxidative stress and cell proliferation. J Neurosci. 2006;26(41):10514-23.

51. Wilson CB, McLaughlin LD, Nair A, Ebenezer PJ, Dange R, Francis J. Inflammation and oxidative stress are elevated in the brain, blood, and adrenal glands during the progression of post-traumatic stress disorder in a predator exposure animal model. PLoS ONE. 2013;8(10):e76146.

52. Hassan W, de Castro GV, Pinton S, da Rocha JBT, Landeira-Fernandez J. Association between oxidative stress and contextual fear conditioning in Carioca high-and low-conditioned freezing rats. Brain Res. 2013;1512:60-7.

53. Ferrero E, Saccucci F, Malavasi F. The human CD38 gene: polymorphism, CpG island, and linkage to the CD157 (BST-1) gene. Immunogenetics. 1999;49(7):597-604.

54. Hirata Y, Kimura N, Sato K, Ohsugi Y, Takasawa S, Okamoto H, et al. ADP ribosyl cyclase activity of a novel bone marrow stromal cell surface molecule, BST-1. FEBS Lett. 1994;356(2-3):244-8.

55. Essuman K, Summers DW, Sasaki Y, Mao X, DiAntonio A, Milbrandt J. The SARM1 Toll/interleukin-1 receptor domain possesses intrinsic NAD+ cleavage activity that promotes pathological axonal degeneration. Neuron. 2017;93(6):1334-43.

56. Zhang H, Graeff R, Lee HC, Hao Q. Crystal structures of human CD38 in complex with NAADP and ADPRP. Messenger. 2013;2(1):44-53.

57. Wei W, Graeff R, Yue J. Roles and mechanisms of the CD38/cyclic adenosine diphosphate ribose/Ca2+ signaling pathway. World J Biol Chem. 2014;5(1):58

58. Kim S, Kim T, Lee H-R, Jang E-H, Ryu H-H, Kang M, et al. Impaired learning and memory in CD38 null mutant mice. Mol Brain. 2016;9(1):1-7.

59. Martucci LL, Amar M, Chaussenot R, Benet G, Bauer O, De Zélicourt A, et al. A multiscale analysis in CD38-/- mice unveils major prefrontal cortex dysfunctions. FASEB J. 2019;33(5):5823-35.

60. Lopatina O, Yoshihara T, Nishimura T, Zhong J, Akther S, Fakhrul AA, et al. Anxiety-and depression-like behavior in mice lacking the CD157/ BST1 gene, a risk factor for Parkinson's disease. Front Behav Neurosci. 2014;8:133

61. Lin C-W, Hsueh Y-P. Sarm1, a neuronal inflammatory regulator, controls social interaction, associative memory and cognitive flexibility in mice. Brain Behav Immun. 2014;37:142-51.

62. Wang $Q$, Wang $Q$, Song X-L, Jiang $Q$, Wu Y-J, Li Y, et al. Fear extinction requires $\mathrm{ASIC} 1 \mathrm{a}$-dependent regulation of hippocampal-prefrontal correlates. Sci Adv. 2018;4(10):eaau3075.

63. Oh TS, Cho H, Cho JH, Yu S-W, Kim E-K. Hypothalamic AMPK-induced autophagy increases food intake by regulating NPY and POMC expression. Autophagy. 2016;12(11):2009-25.

\section{Publisher's Note}

Springer Nature remains neutral with regard to jurisdictional claims in published maps and institutional affiliations. 